\title{
Caracterização ultrassonográfica das estruturas flexoras musculotendíneas e ligamentares da porção distal dos membros de novilhas cruzadas
}

\author{
[Ultrasonographic characterization of flexor tendinous structure muscle and ligaments \\ of the distal limb of crossbred heifers] \\ G.M. Nogueira ${ }^{1}$, J.W. Cattelan ${ }^{2}$, W.A.B. Pereira ${ }^{3}$, P.C. Moraes $^{2}$, C.A. Duarte ${ }^{4}$ \\ ${ }^{1}$ Aluno de pós-graduação - Faculdade de Medicina Veterinária e Zootecnia, \\ Universidade Estadual Paulista "Júlio de Mesquita Filho" - UNESP - Botucatu, SP \\ ${ }^{2}$ Faculdade de Ciências Agrárias e Veterinárias - UNESP - Jaboticabal, SP \\ ${ }^{3}$ Universidade de Uberaba - UNIUBE - Uberaba, MG \\ ${ }^{4}$ Universidade Federal do Pampa - UNIPAMPA - Uruguaiana, RS
}

\begin{abstract}
RESUMO
Caracterizaram-se, por meio da ultrassonografia, as estruturas flexoras da porção distal dos membros de bovinos utilizando-se peças anatômicas da porção distal dos membros torácicos e pélvicos, provenientes de 20 novilhas mestiças da raça Nelore, com idades entre 24 e 36 meses. Para análise ultrassonográfica, foram estabelecidas cinco zonas de avaliação no plano transversal, denominadas, respectivamente, de zonas A, B, C, D e E, e duas em plano sagital, F-III e F-IV. Na face flexora, foram avaliados os tendões flexores digitais superficial e profundo, o músculo interósseo, o ligamento acessório do tendão flexor digital profundo e a manica flexoria, quanto à forma, limites, posição, ecogenicidade e mensurações das áreas transversais em $\mathrm{cm}^{2}$. Sendo os resultados apresentados na forma descritiva e em tabelas, foi possível a caracterização das estruturas flexoras, identificando e determinando planos ultrassonográficos apropriados para a observação de imagens adequadas destes tecidos, além da obtenção de valores e parâmetros que possam ser utilizados como referência para esta espécie.
\end{abstract}

Palavras-chave: ultrassonografia, bovino, estruturas flexoras

\begin{abstract}
Anatomical specimens of the distal portion of the thoracic and pelvic limbs from 20 crossbred Nelore, aged between 24 and 36 months were studied. For ultrasound examination five areas of assessment in the transverse plane were established, respectively called zones $A, B, C, D$ and $E$, and two in the sagittal plane, F-III and F$I V$. On the face of the flexor tendons we assessed superficial and deep digital flexor, interosseous muscle, ligament tendon and deep digital flexor of shape, manica flexoria, boundaries, location, echogenicity and crosscutting areas in $\mathrm{cm}^{2}$, and the results were presented as descriptive and tables. This work was possible to characterize the structures flexor, identifying and determining appropriate plans for the sonographic observation of appropriate images of these tissues, in addition to obtaining values and parameters that can be used as reference for this species, increasing knowledge described in literature.
\end{abstract}

Keywords: ultrasonographic, bovine, flexor structures

\section{INTRODUÇÃO}

A aplicação clínica da ultrassonografia em bovinos tem seu primeiro registro em confirmação de prenhez (Fraser et al., 1971), e sua prática, desde então, enfatiza a área reprodutiva da espécie (Tryon e Clark, 1999).
Em equinos, a avaliação de injúrias musculoesqueléticas por meio do ultrassom é uma realidade desde sua introdução em 1980 (Tryon e Clark, 1999), tornando-se comum sua utilização principalmente no diagnóstico e monitoramento de lesões tendíneas ou ligamentares (Craychee, 1995; Tryon e Clark, 1999).

Recebido em 14 de janeiro de 2011

Aceito em 14 de abril de 2011

E-mail: gmorelnogueira@gmail.com 
Lesões tendíneas são causas reconhecidas de disfunções locomotoras em bovinos, sendo o exame ultrassonográfico um dos métodos auxiliares para diagnóstico de injúrias tendíneas nesta espécie (Anderson e St Jean, 1996). As estruturas musculotendíneas de suporte mais importantes da porção distal dos membros de bovinos encontram-se situadas na faces palmar ou plantar. Consistem, principalmente, das inserções tendíneas de três músculos, o flexor digital superficial, o flexor digital profundo e o interósseo, também denominado ligamento suspensório (Tryon e Clark, 1999).

O interesse recente no uso da ultrassonografia na avaliação de afecções digitais, aliado à carência de estudos nacionais sobre o assunto, criou motivação para este estudo. Assim, o objetivo desta pesquisa foi estudar, por meio da ultrassonografia, as estruturas que compõem a face flexora da porção distal dos membros de bovinos, visando acrescentar informações à área da veterinária de estudos por imagem.

\section{MATERIAL E MÉTODOS}

Foram utilizados os membros locomotores torácicos e pélvicos provenientes de 20 novilhas mestiças da raça Nelore, com idades entre 24 e 36 meses, criadas em sistema extensivo. As peças foram obtidas em abatedouro, sob fiscalização do Serviço de Inspeção Oficial.

Foram separadas a partir das articulações radiocárpica (membros torácicos) e tibiotársica (membros pélvicos), identificadas, enumeradas, lavadas em água corrente com auxílio de escova, acondicionadas em recipiente plástico e mantidas sob congelamento $\left(\mathrm{a}-18^{\circ} \mathrm{C}\right)$ até o início da fase experimental. Previamente à execução da fase experimental, cada membro foi submetido a descongelamento e tricotomizado nas faces palmar ou plantar, lateral e medial, desde as articulações radiocárpica e tibiotársica até a região coronária dos dígitos.

Foi utilizado o aparelho de raio X (CRX, modelo 500/125-DM), para a realização de exames radiográficos em todos os membros, previamente à investigação ultrassonográfica. Na execução das radiografias, padronizou-se a técnica radiográfica em $55 \mathrm{kVp}, 4 \mathrm{mAs}$, distância focofilme de $100 \mathrm{~cm}$, sem utilização de grade, usando-se filme radiográfico Kodak ${ }^{\circledR}$, tamanho $30 \times 40 \mathrm{~cm}$, usando-se os posicionamentos lateromedial e dorsopalmar ou dorsoplantar dos membros. As imagens ultrassonográficas foram obtidas com a utilização de aparelho de ultrassom semiportátil (Honda, modelo HS 2000), com transdutor linear e frequência de $10 \mathrm{MHz}$, sem o auxílio de almofada de silicone acoplada. A sequência usada nos exames ultrassonográficos foi padronizada, iniciando-se da região proximal para a distal, independente do membro, utilizando-se gel de contato como meio anulador de impedância acústica.

O estudo das estruturas flexoras incluiu o tendão flexor digital superficial (TFDS), o tendão flexor digital profundo (TFDP), o músculo interósseo (MI), o ligamento acessório do tendão flexor digital profundo (LA) e a manica flexoria (MF), das regiões metacárpica e metatársica, até a zona queratógena (talão).

O estudo foi realizado a partir da divisão dos membros em sete regiões, sendo cinco demarcadas no plano transversal e duas no plano sagital, denominadas, respectivamente, de zonas A, B, C, D, E e F-III e F-IV. Estas áreas foram identificadas segundo critérios obtidos a partir da anatomia ultrassonográfica distal dos membros, utilizando-se pontos de melhor visibilização individual de cada estrutura, adaptando-se dos utilizados por Tryon e Clark (1999) em bovinos.

As cinco zonas estabelecidas no plano transversal foram: zona $\mathrm{A}$, referente ao terço médio das regiões metacarpiana ou metatarsiana; zona $\mathrm{B}$, na porção proximal do terço distal das regiões metacarpiana ou metatarsiana, com evidência do início de divisão completa do TFDP; zona $\mathrm{C}$, imediatamente proximal às articulações metacarpofalangiana ou metatarsofalangiana, correspondente ao início da bifurcação do TFDS; zona D, nas articulações metacarpofalangiana ou metatarsofalangiana, em local coincidente com a presença dos ossos sesamoides proximais e a formação da manica flexoria; zona E, entre os paradígitos e o casco.

No plano sagital, foram determinadas as zonas FIII e F-IV, correspondentes às porções distais das regiões metacarpiana ou metatarsiana, demarcadas distalmente pelos ossos sesamoides proximais, sendo a zona F-III obtida no plano sagital medial e a F-IV no plano sagital lateral em relação ao membro, sobrepondo-se aos ramos abaxial e axial dos tendões flexores digitais. 
Com o transdutor orientado no plano transversal, foram feitas as mensurações e as avaliações das imagens das zonas A, B, C, D e E quanto à forma, limites, posição, ecogenicidade e áreas transversais das estruturas anatômicas identificadas. Para a determinação das áreas transversais (AT), foi utilizado programa computacional de análise de imagens Eview ${ }^{\circledR}$ (Echo Image Viewer), obtendo-se as mensurações em $\mathrm{cm}^{2}$. Orientando-se o transdutor no plano sagital em relação aos membros, avaliaram-se as zonas F-III e F-IV quanto aos limites, posição, ecogenicidade e disposição (orientação) das fibras musculares e tendíneas.

Realizou-se análise de variância, seguida de comparação de médias pelo teste Tukey, com nível de significância de 5\%, utilizando-se o programa "Statistica" (Statsoft ${ }^{\circledR}$ ). As medidas obtidas das AT foram analisadas em relação aos ramos mediais e laterais das estruturas no mesmo membro, em comparações ipsilateral, contralateral e entre os mesmos membros (torácicos e pélvicos).

\section{RESULTADOS E DISCUSSÃO}

Os achados obtidos no exame ultrassonográfico são apresentados de forma descritiva para cada estrutura anatômica isoladamente e, na sequência, encontram-se expressas as mensurações obtidas (em $\left.\mathrm{cm}^{2}\right)$ nas áreas transversais (Tab. 1 a 5).

Na zona A, o TFDS apresenta-se em forma de meia-lua (Fig. 1), limitado pela pele e faces palmar ou plantar do tendão do músculo flexor digital profundo, e está posicionado mais superficialmente em relação às demais estruturas. Observa-se ecogenicidade intermediária quando comparado ao TFDP e ao músculo interósseo, e é contornado por linha hiperecoica em padrão variado, presente ao redor de toda a estrutura, ou apenas parcialmente em seus limites palmar (plantar) ou dorsal.

Na zona B, o TFDS permanece com formato de meia-lua (Fig. 2), excetuando-se o fato de apresentar, por vezes, uma proeminência no terço médio da face dorsal, onde, posteriormente, ocorrerá sua bifurcação. São mantidas as características de limites e posicionamento da zona anterior, e correlaciona-se com as faces palmar ou plantar dos tendões do músculo flexor digital profundo, agora completamente dividido em dois ramos. Apresenta-se isoecoico, quando comparado ao TFDP e ao LA, com contornos hiperecoicos variados.

Na zona C, em evidente divisão em dois ramos, ambos em formato de meia-lua, o TFDS mantém-se limitado pelo tegumento e as faces palmar ou plantar dos ramos do TFDP (Fig. 3). Posicionado mais superficialmente, apresenta-se ainda isoecoico quando comparado ao TFDP e ao LA.

Na zona D, não foi possível visibilizar o TFDS, pelo fato de ele constituir, neste momento, o limite dorsal da manica flexoria (Fig. 4).

Na zona E, o TFDS apresenta-se achatado nos sentidos dorsopalmar ou dorsoplantar (Fig. 5). Seus ramos são limitados pelas faces dorsais dos ramos do TFDP e pelas faces palmar ou plantar dos ligamentos palmares ou plantares da articulação interfalangiana proximal. Posicionase de forma invertida em relação aos ramos do TFDP e apresenta contornos irregulares em ecogenicidade, com a presença de pequenas áreas anecoicas ou hipoecoicas circulares, variando em número, diâmetro e localização, próximas às bordas do tendão.

Nas zonas F-III e F-IV, limitado pelas faces palmar ou plantar do TFDP e pela pele, o TFDS é a estrutura mais superficial (Fig. 6). Posicionado palmar ou plantarmente ao TFDP, apresenta linhas hiperecoicas em seus limites dorsal e palmar ou plantar, além de alterações de ecogenicidade em sua extensão, visibilizadas por variações nas escalas de cinza. Apresenta arranjo linear das fibras tendíneas, dispostas horizontalmente. 


\section{Caracterização ultrassonográfica...}

Tabela 1. Médias e desvios-padrão de áreas transversais (AT), em $\mathrm{cm}^{2}$, obtidos nas mensurações dos tendões flexores digitais superficiais dos membros de bovinos

\begin{tabular}{lllll}
\hline TFDS & MPD & MPE & MTD & MTE \\
\hline Zona A & $3,15 \pm 0,39$ & $3,15 \pm 0,54$ & $3,11 \pm 0,44$ & $3,20 \pm 0,36$ \\
Zona B & $4,05 \pm 0,75$ & $3,95 \pm 0,68$ & $3,95 \pm 0,60$ & $4,08 \pm 0,77$ \\
Zona C - Axial & $2,25 \pm 0,77$ & $2,16 \pm 0,39$ & $2,58 \pm 0,62$ & $2,40 \pm 0,40$ \\
Zona C - Abaxial & $2,43 \pm 0,69$ & $2,31 \pm 0,40$ & $2,24 \pm 0,52$ & $2,11 \pm 0,38$ \\
Zona E - Axial & $1,88 \pm 0,43$ & $1,76 \pm 0,30$ & $1,92 \pm 0,44$ & $1,85 \pm 0,29$ \\
Zona E - Abaxial & $2,08 a b \pm 0,37$ & $1,94 \pm 0,27$ & $1,72 \mathrm{a} \pm 0,44$ & $1,65 \mathrm{~b} \pm 0,27$ \\
\hline
\end{tabular}

${ }^{a}$ Indica diferenças $(\mathrm{p} \leq 0,05)$ pelo teste Tukey, nas comparações entre os membros torácicos e pélvicos ipsilaterais.

${ }^{b}$ Indica diferenças $(\mathrm{p} \leq 0,05)$ pelo teste Tukey, nas comparações entre os membros torácicos e pélvicos contralaterais.

TFDS= tendão flexor digital profundo; $\mathrm{MPD}=$ membro pélvico direito; $\mathrm{MPE}=$ membro pélvico esquerdo; $\mathrm{MTD}=$ membro torácico direito; MTE= membro torácico esquerdo.

Tabela 2. Médias e desvios-padrão de áreas transversais (AT), em $\mathrm{cm}^{2}$, obtidos nas mensurações dos tendões flexores digitais profundos dos membros de bovinos

\begin{tabular}{lllll}
\hline TFDP & MPD & MPE & MTD & MTE \\
\hline Zona A & $3,54 \pm 0,35$ & $3,61 \pm 0,45$ & $3,69 \pm 0,34$ & $3,71 \pm 0,60$ \\
Zona B - Axial & $2,50 \pm 0,49$ & $2,55 \pm 0,62$ & $2,73 \pm 0,44$ & $2,87 \pm 0,67$ \\
Zona B - Abaxial & $2,87 \pm 0,62$ & $2,88 \pm 0,68$ & $2,51 \pm 0,56$ & $2,59 \pm 0,75$ \\
Zona C - Axial & $3,04 a \pm 0,55$ & $3,10 \pm 0,39$ & $3,56 a \pm 0,56$ & $3,44 \pm 0,51$ \\
Zona C - Abaxial & $3,49 \pm 0,46$ & $3,34 \pm 0,41$ & $3,23 \pm 0,54$ & $3,12 \pm 0,62$ \\
Zona D - Axial & $2,73 \pm 0,54$ & $2,83 \pm 0,61$ & $2,95 \pm 0,59$ & $3,05 \pm 0,58$ \\
Zona D - Abaxial & $2,96 \pm 0,58$ & $3,01 \pm 0,61$ & $2,56 \pm 0,48$ & $2,66 \pm 0,61$ \\
Zona E - Axial & $2,69 \pm 0,42$ & $2,50 \pm 0,52$ & $2,75 \pm 0,52$ & $2,70 \pm 0,52$ \\
Zona E - Abaxial & $3,03 a b \pm 0,63$ & $2,90 a \pm 0,64$ & $2,47 a \pm 0,62$ & $2,37 \mathrm{ab} \pm 0,47$ \\
\hline
\end{tabular}

${ }^{a}$ Indica diferenças $(\mathrm{p} \leq 0,05)$ pelo teste Tukey nas comparações entre os membros torácicos e pélvicos ipsilaterais.

${ }^{\mathrm{b}}$ Indica diferenças $(\mathrm{p} \leq 0,05)$ pelo teste Tukey nas comparações entre os membros torácicos e pélvicos contralaterais.

TFDS= tendão flexor digital profundo; $\mathrm{MPD}=$ membro pélvico direito; $\mathrm{MPE}=$ membro pélvico esquerdo; $\mathrm{MTD}=\mathrm{membro}$ torácico direito; MTE= membro torácico esquerdo.

Tabela 3. Médias e desvios-padrão de áreas transversais (AT), em $\mathrm{cm}^{2}$, obtidos nas mensurações dos músculos interósseos dos membros de bovinos

\begin{tabular}{lllll}
\hline MI & MPD & MPE & MTD & MTE \\
\hline Zona A & $3,33 \pm 0,48$ & $3,24 a \pm 0,48$ & $3,62 \pm 0,64$ & $3,73 a \pm 0,42$ \\
Zona B - Axial & $2,41 \pm 0,46$ & $2,31 \pm 0,34$ & $2,51 \pm 0,43$ & $2,68 \pm 0,45$ \\
Zona B - Abaxial & $2,69 \pm 0,40$ & $2,66 \pm 0,43$ & $2,30 \pm 0,44$ & $2,38 \pm 0,50$ \\
Zona C - Axial & $2,75 \pm 0,49$ & $2,62 \pm 0,42$ & $2,89 \pm 0,56$ & $2,93 \pm 0,51$ \\
Zona C - Abaxial & $2,78 \pm 0,47$ & $2,67 \pm 0,42$ & $2,79 \pm 0,55$ & $2,70 \pm 0,49$ \\
\hline
\end{tabular}

${ }^{a}$ Indica diferenças $(\mathrm{p} \leq 0,05)$ pelo teste Tukey nas comparações entre os membros torácicos e pélvicos ipsilaterais.

$\mathrm{MI}=$ músculo interósseo; $\mathrm{MPD}=$ membro pélvico direito; $\mathrm{MPE}=$ membro pélvico esquerdo; $\mathrm{MTD}=$ membro torácico direito; $\mathrm{MTE}=$ membro torácico esquerdo.

Tabela 4. Médias e desvios-padrão de áreas transversais (AT), em $\mathrm{cm}^{2}$, obtidos nas mensurações dos ligamentos acessórios dos tendões flexores digitais profundos dos membros de bovinos

\begin{tabular}{lllll}
\hline LA & MPD & MPE & MTD & MTE \\
\hline Zona A & $2,65 \pm 0,54$ & $2,50 \pm 0,48$ & $2,80 \pm 0,75$ & $2,70 \pm 0,52$ \\
Zona B - Axial & $1,76 \mathrm{~b} \pm 0,53$ & $1,80 \mathrm{a} \pm 0,51$ & $2,07 \pm 0,50$ & $2,28 \mathrm{ab} \pm 0,50$ \\
Zona B - Abaxial & $1,95 \pm 0,47$ & $2,03 \pm 0,56$ & $1,90 \pm 0,45$ & $2,03 \pm 0,42$ \\
Zona C - Axial & $2,08 \pm 0,73$ & $2,05 \pm 0,54$ & $2,28 \pm 0,50$ & $2,36 \pm 0,63$ \\
Zona C - Abaxial & $2,31 \pm 0,71$ & $2,26 \pm 0,70$ & $2,02 \pm 0,43$ & $2,04 \pm 0,58$ \\
\hline
\end{tabular}

${ }^{a}$ Indica diferenças $(\mathrm{p} \leq 0,05)$ pelo teste Tukey nas comparações entre os membros torácicos e pélvicos ipsilaterais.

${ }^{b}$ Indica diferenças $(\mathrm{p} \leq 0,05)$ pelo teste Tukey nas comparações entre os membros torácicos e pélvicos contralaterais.

$\mathrm{LA}=$ ligamento acessório; $\mathrm{MPD}=$ membro pélvico direito; $\mathrm{MPE}=$ membro pélvico esquerdo; $\mathrm{MTD}=$ membro torácico direito; MTE= membro torácico esquerdo.

Tabela 5. Médias e desvios-padrão de áreas transversais (AT), em $\mathrm{cm}^{2}$, obtidos nas mensurações da manica flexoria dos membros de bovinos

\begin{tabular}{lllll}
\hline MF & MPD & MPE & MTD & MTE \\
\hline Zona D - Axial & $5,16 \pm 0,72$ & $5,10 \pm 0,83$ & $5,29 \pm 0,70$ & $5,22 \pm 0,86$ \\
Zona D - Abaxial & $5,46 \pm 0,75$ & $5,45 \pm 0,83$ & $4,93 \pm 0,73$ & $4,94 \pm 0,82$ \\
\hline
\end{tabular}

Não houve diferenças ( $>00,05)$ pelo teste Tukey nas comparações entre os membros torácicos e pélvicos.

$\mathrm{MF}=$ manica flexoria; $\mathrm{MPD}=$ membro pélvico direito; $\mathrm{MPE}=$ membro pélvico esquerdo; $\mathrm{MTD}=$ membro torácico direito; $\mathrm{MTE}=$ membro torácico esquerdo. 


\section{Nogueira et al.}
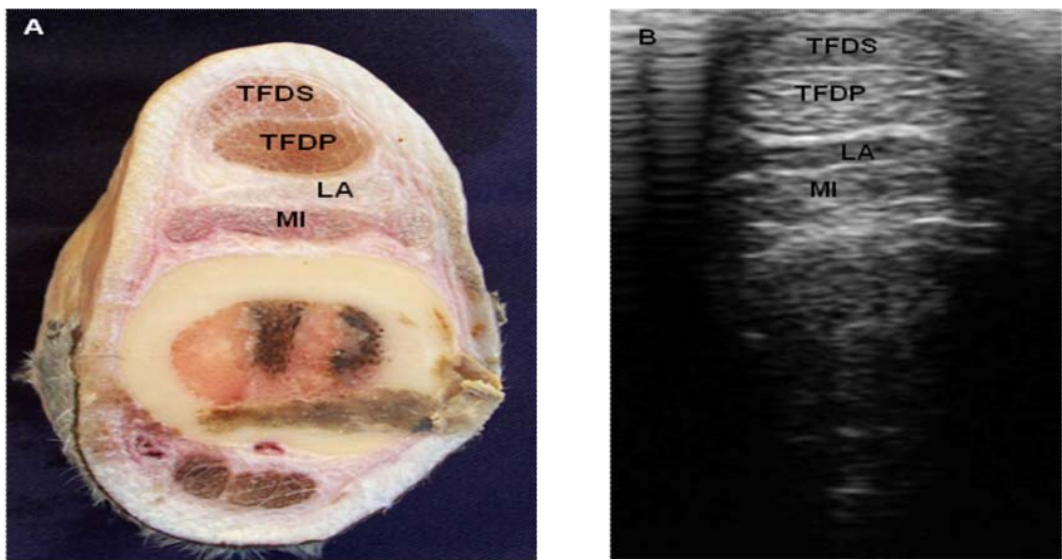

Figura 1. Ilustrações macroscópica (A) e ultrassonográfica (B) do tendão flexor digital profundo (TFDP) do membro de novilha mestiça da raça Nelore, na zona A de avaliação ultrassonográfica, em plano transversal. (TFDS: tendão flexor digital superficial; LA: ligamento acessório do tendão flexor digital profundo; MI: músculo interósseo).
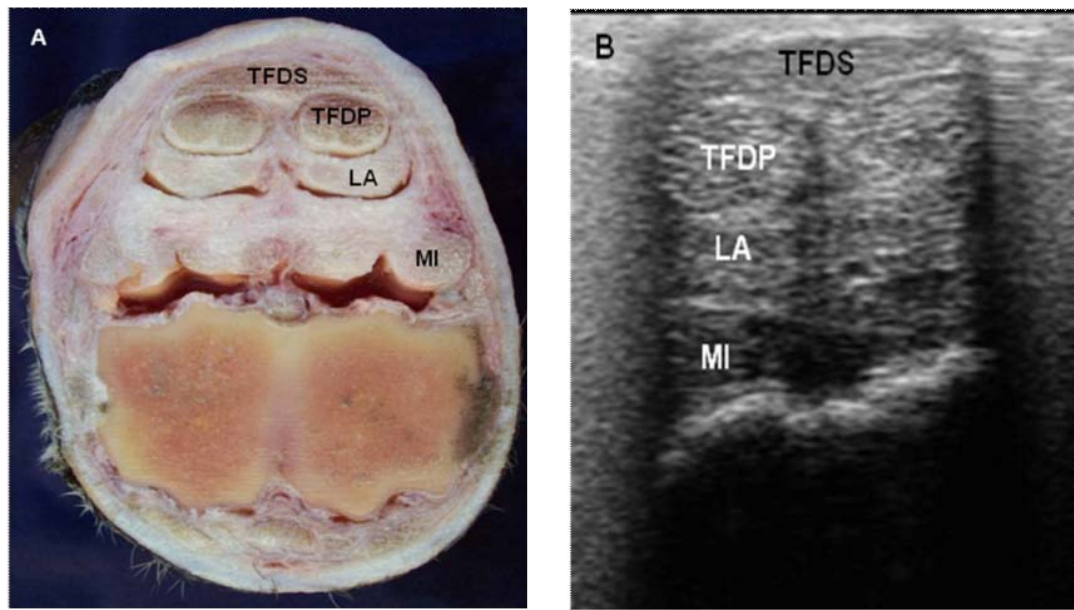

Figura 2. Ilustrações macroscópica (A) e ultrassonográfica (B) do tendão flexor digital profundo (TFDP) do membro de novilha mestiça da raça Nelore, na zona B de avaliação ultrassonográfica, em plano transversal. (TFDS: tendão flexor digital superficial; LA: ligamento acessório do tendão flexor digital profundo; MI: músculo interósseo).
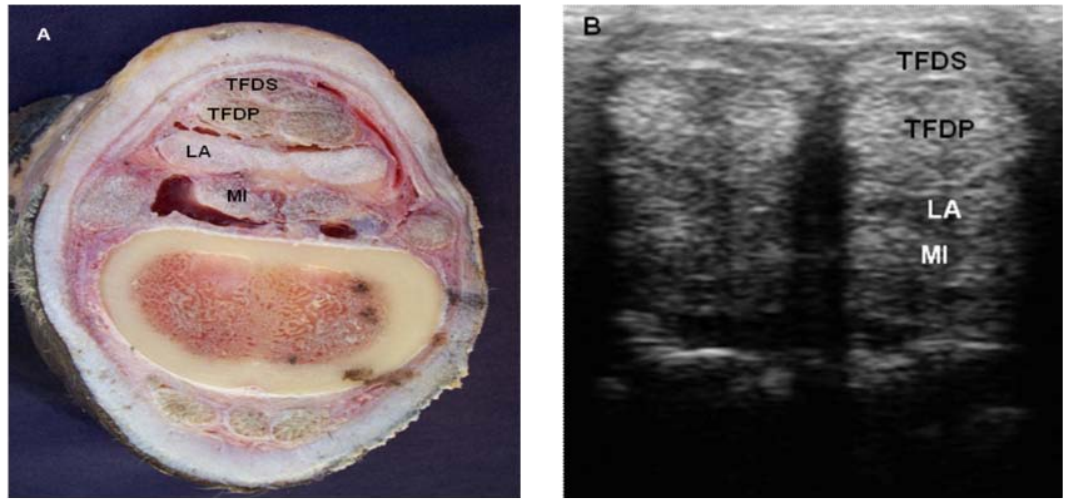

Figura 3. Ilustrações macroscópica (A) e ultrassonográfica (B) do tendão flexor digital profundo (TFDP) do membro de novilha mestiça da raça Nelore, na zona $\mathrm{C}$ de avaliação ultrassonográfica, em plano transversal. (TFDS: tendão flexor digital superficial; LA: ligamento acessório do tendão flexor digital profundo; MI: músculo interósseo). 


\section{Caracterização ultrassonográfica...}
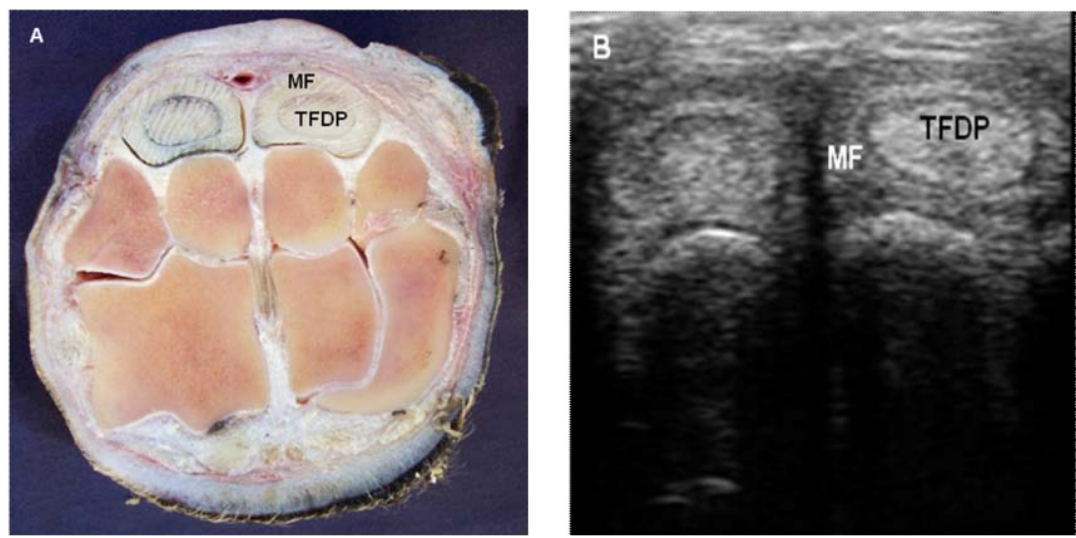

Figura 4. Ilustrações macroscópica (A) e ultrassonográfica (B) do tendão flexor digital profundo (TFDP) do membro de novilha mestiça da raça Nelore, na zona D de avaliação ultrassonográfica, em plano transversal. (MF: manica flexoria).
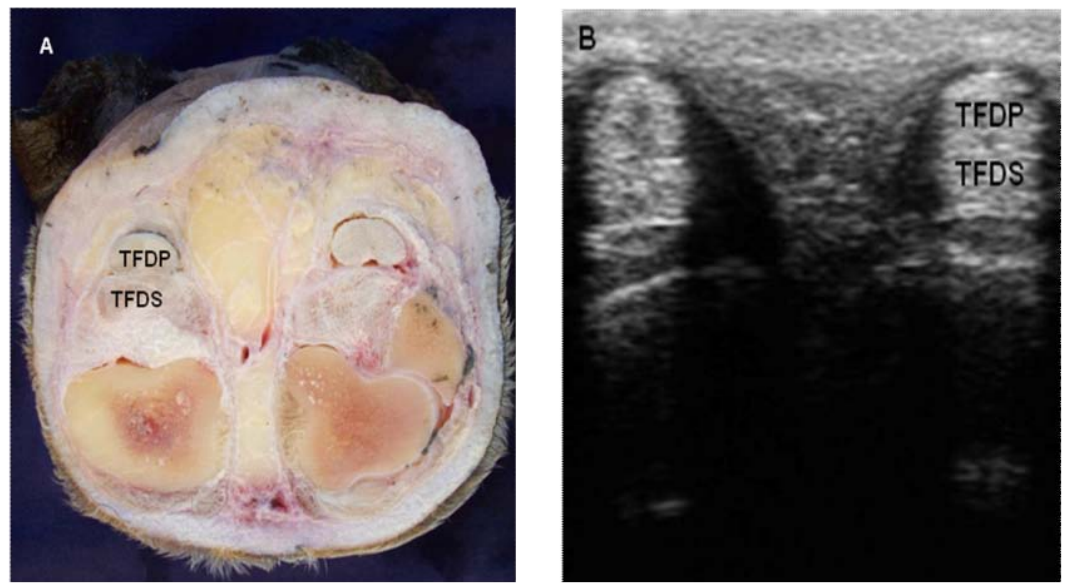

Figura 5. Ilustrações macroscópica (A) e ultrassonográfica (B) do tendão flexor digital profundo (TFDP) do membro de novilha mestiça da raça Nelore, na zona E de avaliação ultrassonográfica, em plano transversal. (TFDS: tendão flexor digital superficial).
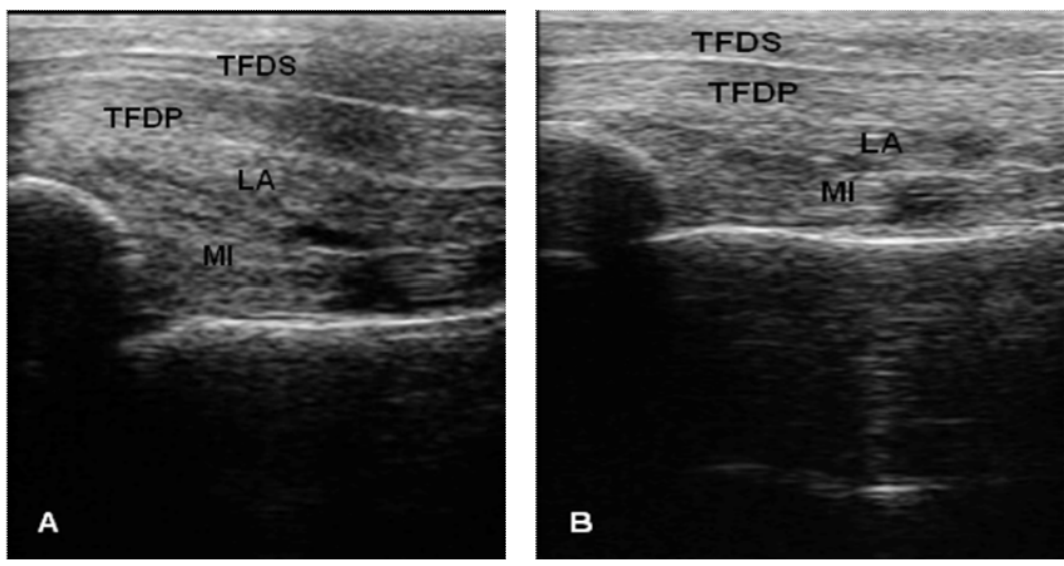

Figura 6. Ilustrações ultrassonográficas do tendão flexor digital profundo (TFDP) do membro de novilha mestiça da raça Nelore, nas zonas F-III (A) e F-IV (B) de avaliação ultrassonográfica, em plano longitudinal. (TFDS: tendão flexor digital superficial; LA: ligamento acessório do tendão flexor digital profundo; MI: músculo interósseo). 
Na zona A, o TFDP apresenta-se em forma arredondada, achatado dorsoventralmente (Fig. 1). É perceptivelmente mais espesso que o TFDS, com contornos regulares. Limitado pelas faces dorsal do TFDS e palmar ou plantar do ligamento acessório do tendão flexor digital profundo, posiciona-se dorsalmente ao TFDS. É contornado por uma linha hiperecoica em seus limites dorsal e palmar ou plantar, com padrão homogêneo de ecogenicidade.

Na zona B, a partir desta zona e servindo como delimitação para o seu início, o TFDP apresenta divisão completa em dois ramos, em forma circular (Fig. 2). É limitado pela face dorsal do tendão único do músculo flexor digital superficial e pelas faces palmar ou plantar dos ramos do ligamento acessório do tendão flexor digital profundo, posicionando-se entre estas estruturas. Apresenta padrão homogêneo de ecogenicidade, podendo ser visibilizadas, por vezes, áreas irregulares com padrão hipoecoico em seu interior, de localização e extensão variadas.

Na zona C, a forma do TFDP varia de circular a levemente achatada dorsopalmarmente ou dorsoplantarmente. Este se apresenta limitado pelas faces dorsais do TFDS, completamente dividido em dois ramos (Fig. 3), e pelas faces palmar ou plantar do ligamento acessório do tendão flexor digital profundo, posicionando-se dorsalmente ao TFDS. Possui padrão homogêneo de ecogenicidade, podendo ser visibilizados dois núcleos ovalados mais ecogênicos no interior de cada ramo do TFDP.

Na zona D, embora envolto por uma estrutura tubular, a manica flexoria, o TFDP apresenta forma arredondada, com leve achatamento dorsopalmar ou dorsoplantar (Fig. 4). Limitado pela manica flexoria, encontra-se posicionado no interior desta. Apresenta variações de ecogenicidade, contudo, mais ecogênico que a manica flexoria, notando-se áreas hipoecoicas no interior do TFDP, que variam em extensão e localização.

Na zona E, o TFDP é visibilizado como dois ramos circulares digitais (III e IV), limitados pelo tegumento e pelas faces palmar ou plantar do TFDS. Tais ramos estão localizados mais superficialmente, em posicionamento invertido com os ramos do TFDS (Fig. 5). Observa-se linha hiperecoica no limite dorsal e de área anecoica ao redor das estruturas visibilizadas. Há padrão heterogêneo de ecogenicidade, evidenciado pelas variações na tonalidade da escala de cor cinza.

Nas zonas F-III e F-IV, o TFDP, limitado pelas faces dorsal do TFDS e palmar ou plantar do ligamento acessório do tendão flexor digital profundo, encontra-se posicionado dorsalmente ao TFDS (Fig. 6). Apresenta-se mais ecogênico que o TFDS, o LA e o MI, sendo demarcado nas faces palmar ou plantar por linhas hiperecoicas. Observa-se arranjo de fibras lineares, dispostas horizontalmente

Na zona A, o MI apresenta forma semelhante à retangular, com bordas arredondadas (Fig. 1). Está limitado pela face dorsal do ligamento acessório do tendão flexor digital profundo e pelas faces palmar ou plantar dos metacarpianos ou metatarsianos III e IV. Posiciona-se dorsalmente ao ligamento acessório do tendão flexor digital profundo e apresenta contornos ecogênicos irregulares, com variadas formações anecoicas ou hipoecoicas visibilizadas no interior.

Na zona B, evidencia-se a divisão do músculo interósseo em quatro ramos (Fig. 2), observados como formas arredondadas, podendo ser visibilizados apenas dois ramos principais. Encontra-se limitado pela face dorsal do ligamento acessório do tendão flexor digital profundo, dividido em dois ramos, e pelas faces palmar ou plantar dos metacarpianos ou metatarsianos III e IV. Posiciona-se dorsalmente aos ramos do ligamento acessório do tendão flexor digital profundo e possui ecogenicidade variada, com padrão heterogêneo evidenciado por meio de variações na tonalidade da escala de cor cinza.

Na zona C, observa-se a apresentação do MI em dois ramos principais, de forma irregularmente arredondada, com achatamento dorsopalmar ou dorsoplantar (Fig. 3). O MI está limitado pelas faces dorsais dos ramos do ligamento acessório do tendão flexor digital profundo, e pelas faces palmar ou plantar dos metacarpianos ou metatarsianos III e IV. Posiciona-se dorsalmente ao ligamento acessório do tendão flexor digital profundo, e há presença de variadas áreas anecoicas, ou hipoecoicas, encontradas no interior do músculo, com padrão irregular de ecogenicidade. 
Nas zonas D e E, não há evidenciação do músculo interósseo (Fig. 4 e 5).

Nas zonas F-III e F-IV, o MI está limitado pelas faces palmar ou plantar dos ossos metacarpianos ou metatarsianos III e IV e pela face dorsal do ligamento acessório do TFDP e é observada a inserção do MI nos ossos sesamoides proximais. Posiciona-se dorsalmente ao ligamento acessório do tendão flexor digital profundo (Fig. 6) e apresenta variações de ecogenicidade, evidenciadas por áreas anecoicas e hipoecoicas em sua extensão. Observa-se arranjo de fibras lineares, dispostas horizontalmente, em forma de leque, na inserção aos ossos sesamoides proximais.

Na zona A, o LA é visibilizado como uma estrutura com apresentação delgada e larga, ampla no sentido lateromedial, com contorno e formato irregulares (Fig. 1). Limitado pelas faces dorsal do TFDP e palmar ou plantar do músculo interósseo, posiciona-se dorsalmente ao TFDP. Apresenta-se hipoecoico e observam-se a presença de linha hiperecoica nos limites dorsal e palmar ou plantar e de variadas áreas circulares anecoicas ou hipoecoicas, no interior do ligamento.

Na zona B, o LA apresenta-se delgado, mais amplo no sentido lateromedial, em forma irregular. Observa-se divisão da estrutura em dois ramos, acompanhando o TFDP. Limitado pelas faces dorsais dos ramos do TFDP e palmar ou plantar do músculo interósseo, posiciona-se dorsalmente ao TFDP (Fig. 2). Observa-se estrutura hipoecoica, com contornos de definição pouco nítida.

Na zona C, o LA apresenta divisão evidente em dois ramos, em formas arredondadas e achatados nos sentidos dorsopalmar ou dorsoplantar (Fig. 3). Limitado pelas faces dorsais dos ramos do TFDP e palmar ou plantar do músculo interósseo, posiciona-se dorsalmente ao TFDP. Hipoecoico e com contornos pouco definidos, o LA apresenta ecotextura irregular nesta região.

Na zona D, compondo o limite dorsal da manica flexoria, o LA encontra-se ausente (Fig. 4).

Na zona E, não há evidenciação do ligamento acessório do TFDP (Fig. 5).
Na zonas F-III e F-IV, limitado pelas faces dorsal do TFDP e palmar ou plantar do músculo interósseo, o LA posiciona-se dorsalmente ao TFDP (Fig. 6). Observa-se estrutura hipoecoica com ecotextura irregular e variações de áreas anecoicas e hipoecoicas em sua extensão. Apresenta arranjo de fibras lineares dispostas horizontalmente.

Nas zonas A, B, C, E, F-III e F-IV, não há evidenciação ultrassonográfica da manica flexoria.

Na zona D, a MF apresenta-se como uma estrutura tubular, formando um anel ao redor do TFDP (Fig. 4). Limitada externamente pelos ossos sesamoides proximais e pela pele, e internamente pelo TFDP, posiciona-se sobre as superfícies palmar ou plantar dos ossos sesamoides proximais. Apresenta ecotextura homogênea e contornos interno e externo facilmente evidenciados.

Durante a avaliação ultrassonográfica, não foram observadas injúrias tendíneas ou ligamentares. Tal avaliação foi difícil de ser feita, frente à existência de artefatos de técnica produzidos durante o exame, tendo em vista que a avaliação ultrassonográfica foi feita em peças anatômicas, onde a ausência de tensão nos tendões, que seria produzida pela sustentação de peso do animal, gerou o relaxamento dos tecidos moles, devido à transecção dos membros nas articulações do carpo e tarso. Isso produziu variações nos padrões de ecogenicidade e ecotextura, sendo evidenciadas como artefatos hipoecoicos, representados por alterações na graduação da escala de cor cinza observadas nas estruturas, conforme já relatado por Wrigley (2006).

Complementarmente, observou-se que a ausência de fluxo sanguíneo originou a presença de outros artefatos de técnica, em que a formação de áreas anecoicas circulares podia ser interpretada como acúmulo de líquido nos tecidos, ou presença de vaso sanguíneo no local examinado. Tal ocorrência foi observada principalmente nas avaliações ultrassonográficas do músculo interósseo e do ligamento acessório do tendão flexor digital profundo, não sendo possível atribuir ao padrão de imagem observado a existência de lesões musculotendíneas. 
Tryon e Clark (1999) descreveram a interpretação errônea de estruturas vasculares em peças anatômicas como acúmulo de fluido no tecido avaliado. Isto porque a visibilização destas estruturas, em animais vivos, ocorre como imagens circulares anecoicas, em plano sagital, concomitante à pulsação frequente visibilizada.

Em bovinos, a mensuração da área transversal ainda não foi descrita na literatura consultada, contudo, à semelhança do que já foi descrito em equinos (Wrigley, 2006), pode fornecer informação fundamental na determinação da taxa de gravidade de lesões tendíneas, assim como dos aumentos percentuais da estrutura tendínea examinada. Convém ressaltar que, em equinos, aumentos de áreas transversais da estrutura tendínea da ordem de $20 \%$ já indicam a ocorrência de lesão (Smith et al., 1994; Wrigley, 2006).

\section{CONCLUSÕES}

A caracterização ultrassonográfica de estruturas musculotendíneas e ligamentares importantes que compõem a face flexora da porção distal dos membros, de forma ordenada, seriada e sequencial, conforme as sete zonas de avaliação preestabelecidas, é exequível. Com o uso desta técnica, é possível realizar a descrição estrutural das imagens visibilizadas mediante a determinação de forma, limites, posicionamento e ecogenicidade, além de mensurações das áreas transversais das estruturas anatômicas. Assim, as informações obtidas por este modo de investigação ultrassonográfica podem fornecer subsídios importantes à buiatria, especialmente no que concerne a diagnósticos e prognósticos de afecções locomotoras sediadas na face flexora das porções distais dos membros de bovinos.

\section{REFERÊNCIAS BIBLIOGRÁFICAS}

ANDERSON, D. E.; ST JEAN, G. Diagnosis and management of tendon disorders in cattle. Vet. Clin. N. Am.: Food Anim. Pract., v.12, p.85-116, 1996.

CRAYCHEE, T.J. Ultrasonographic evaluation of equine musculoskeletal injury. In: NYLAND, T.G.; MATTON, J.S. Veterinary diagnostic ultrasound. Philadelphia: W. B. Saunders, 1995. p.265-304.

FRASER, A.F.; NAGARATNAM, V.; CALLICOTT, R.B. The comprehensive use of doppler ultra-sound in farm animal reproduction. Vet. Rec., v.88, p.202-205, 1971.

SMITH, R.K.W.; JONES, R.; WEBBON, P.M. The cross-sectional areas of normal equine digital flexor tendons determined ultrasonographically. Equine Vet. J., v.26, p.460-465, 1994.

TRYON, K.A.; CLARK, C.R. Ultrasonographic examination of the distal limb of cattle. Vet. Clin. N. Am.: Food Anim. Pract., v.15, p.275-300, 1999.

WRIGLEY, R.H. Ultrassonografia de tendões, ligamentos e articulações. In: STASHAK, T.S. Claudicação em equinos segundo Adams. São Paulo: Roca, 2006. p. 278-310. 